\title{
Boundary Crossings
}

\section{Mixed methods in land change research: towards integration}

\author{
So-Min Cheong*, Daniel G Brown ${ }^{* *}$, Kasper Kok $\dagger$ and \\ David Lopez-Carr
}

\author{
*Department of Geography, University of Kansas, Lawrence, KS 66045, USA \\ email: somin@ku.edu \\ **School of Environment, University of Michigan, Ann Arbor, MI 48710, USA \\ †WU Environmental Science, 6700AA, Wageningen, The Netherlands \\ \$Department of Geography, University of Santa Barbara, Santa Barbara, CA 93106, USA \\ revised manuscript received 8 August 2011
}

\section{Introduction}

Land change science has been strongly influenced by geographers working in geographic information science (Gutman et al. 2004). These geographers typically employ GIS and remote sensing technology to determine the type and magnitude of natural- and human-induced changes on the landscape, focusing on changes in land cover (i.e. the biophysical aspects of land dynamics). As it has matured, however, land change science has moved toward inclusion of themes from geography's humanenvironment tradition and other interdisciplinary endeavours to link natural and human systems in order to understand the human effects and implications of land change (Rindfuss et al. 2004; Moran and Ostrom 2005). Land change science has therefore emerged as an integrative science that attempts to bridge understandings of both land-use (i.e. human and social aspects of land dynamics) and land-cover change as two components of a coupled natural and human system. This new approach has emphasised stronger inclusion of the human processes to establish human-environment interaction methods and models instead of approaches that conceive of human-environment interaction in uni-directional terms, i.e. as human impact on environment as represented in land cover.

Methodologically, the challenge remains to suitably integrate diverse datasets and approaches. Mixed methods ranging from participant observa- tion, interviews, GIS, remote sensing, statistics and computation are used to generate explanation and predictive and representative models and plausible scenarios in land change science (Dearing et al. 2006; Gimblett et al. 2001, Parker et al. 2003; Robinson et al. 2007). This paper considers the question of the appropriate balance between (1) quantitative and qualitative approaches, (2) simulation and observation, and (3) diversity and integration central to the pursuit of an integrative approach to mixed methods in land change science. The paper discusses why the current use of mixed methods remains complementary rather than integrative by addressing gaps and possible solutions. It employs examples primarily from land use, agent-based modelling and scenario development as they reflect some of the latest developments that link social dynamics of land use with land cover. The examples are by no means exhaustive, and they mainly serve to illustrate the main question of appropriate balance.

\section{Quantitative and qualitative methods}

How are we integrating quantitative and qualitative methods in land change research?

One of the most pressing methodological conundrums in land change research is the appropriate integration of quantitative and qualitative information for developing process-based knowledge of land-use dynamics for use in modelling and scenarios. While integration in the study of land 
change has been accomplished by combining data derived from remotely sensed imagery and census or household survey data to infer the connections between land use and land cover, several studies have employed field interviews and ethnographic techniques to gather more explicit land-use data and better understand land-cover change seen in satellite imagery (Lambin and Meyfroidt 2010).

Household and community surveys involve quantitative research with an advantage of sampling a large number of households and communities, and ethnographic methods offer more intensive and nuanced qualitative data than is generally possible with fixed-format questionnaires. They are complementary in that quantitative surveys document the patterns of land use, and qualitative data provide context, validity and explanations to the documented patterns. Household-level land-use decisions important in understanding migration, for example, necessitate the use of participant observation and interviews to apprehend why households choose to migrate to an agricultural frontier (Carr 2008). This is a crucial question for understanding why land cover changes and how the change affects the livelihoods of both residents and new migrants reflected in land-use strategies.

With the rise of the systemic view in the recent turn to human-environment interactions in land change, researchers go beyond combining qualitative and quantitative methods witnessed in landuse research, and actively pursue the integration of both (An et al. 2005). Agent-based models exert a strong influence in this respect, and are particularly suited to modelling complex human behaviour and facilitating decisionmaking ( $\mathrm{D}^{\prime}$ Aquino et al. 2003). They represent the decisions of various agents in the form of computer code. Role-playing games, field experiments, participant observation and surveys can inform modellers what actions agents would take in different situations and modellers attempt to represent those actions as rules or algorithms that can be coded (Robinson et al. 2007). Land change projects tend to use one of these methods exclusively, rather than employing multiple methods within a fully integrated framework, though opportunities exist to use several multiple methods (Smajgl et al. 2011).

As quantitative input is frequently required for agent-based and other land change models, the translation and/or interpretation of qualitative information are often necessary. In the conversion to quantitative input, a holistic and nuanced under- standing of the human system provided by qualitative methods can be lost, and some qualitative data cannot be translated. Social scientists often question whether a translation of this sort can adequately represent human decisionmaking and other processes, and suspect that they may inadvertently lead to a reductionist understanding of human behaviour. An approach that avoids the necessity of such a translation is to use qualitative scenarios as narratives side by side with the models. Narrative storylines are effective ways to represent and integrate stakeholder perceptions into a model application without reducing the elaborated qualitative data into a quantified model input. The goal is to develop models and scenarios in close interaction with stakeholders that are involved in all phases of the research (Alcamo 2009).

In summary, an explicit integration of quantitative and qualitative data is limited to data that can be translated easily among quantitative or qualitative types. Co-existence of both qualitative and quantitative data in a complementary format is the norm thus far. Any research question could benefit from both data as long as it involves some measureable quantities and some attempts at explaining causation by human action. In addition, the results of analyses from both types of data inform the interpretations, inferences, and conclusions, and implicitly contribute to the entire process of land change research. To assess the point at which the combined use of qualitative and quantitative data generates the optimal level of research endeavour requires the intuition and experience of the involved research team and an assessment of scientific merits of doing so. Thus, it is considered as much an art as science.

\section{Simulation and observation}

How often do simulated land change models need to be updated with observed data?

The tools of representation and prediction using simulation techniques and traditional field-based observations or lab experiments are not mutually exclusive, in the sense that simulation-based models take inputs from observations and lab experiments. Concerns have been raised about over-reliance on models that diminish the significance and frequency of field-based observations (Pikley and Pilkey-Jarvis 2007). One concern is the accuracy of predictions. Model accuracies are affected by the quality of input data, the nature of the fit of the model to 
those data, and the degree of stationarity in the process itself. The problem of inaccuracies in predictions can be somewhat alleviated by using scenarios that pose as plausible futures and not predictions.

A related issue is the frequency of observation in terms of data collection and monitoring. Pilkey and Pilkey-Jarvis (2007) suggest modelling may offer an easier way out of a lengthy and costly field study. As assumptions based on observations and experience change over time and space, models have difficulty reflecting this in the structure or as a process because models depend on a set of fixed assumptions for a certain period of time at a certain spatial scale. The embedded assumptions render the system open (Oreskes et al. 1994) to be modified and evaluated by frequent field observations and lab experiments. This raises questions about the 'right' frequency and consistency of observational data for evaluation and monitoring.

Iteration between model building and empirical observation is one way to balance simulation and observation. In the case of scenario development, iteration enhances consistency between narrative stories and model outputs. Increasing the number of iterations can be achieved by using an existing set of scenarios (either models or stories or both) or developing meta-models that can be run in real time during a stakeholder workshop. The runtime of the meta-model - which needs to be in the order of seconds rather than minutes - is important as a starting point. Stakeholders are then shown model results and given the opportunity to learn from and comment on them (Guyot and Shinichi 2006). The iterations offer a degree of freedom for stakeholders to develop stories while having access to quantitative information from models.

Increased frequency of observations during and after model formulations can also strengthen model accuracy and predictive power. This suggests that more scientists are needed to work on field-based observations than model simulations that contribute to building a robust model. To this end, the availability of resources and the propensity for adaptive management prove necessary. The role of adaptive management in the context of model development concerns updates on existing models and alternative model testing through system monitoring to the incorporation of experimentation (Norton 2005). Observation frequency can increase data reliability accompanied by modelling that demonstrates the system interactions.

\section{Diversity and integration}

How do we know when integration is sufficiently well conceived and practised?

The tension among diverse paradigms, disciplines, methods and tools exists and influences the success of land change research and relevant policies. Too many methods can overwhelm stakeholders who may consequently become disengaged. Any new method will introduce additional uncertainty. Over-emphasis on diversity can undermine the overarching goal of integrated land change research. Restricting the number of approaches can increase clarity on the type of scientific disciplines to engage and issues to address (Kok et al. 2007).

Different paradigms and diverse data and approaches, for instance, produce inconsistencies between stakeholder perceptions and model outputs in scenario development. Stories tend to emphasise social and institutional change, whereas diagnostic models tend to focus on biophysical and economic change. Sometimes there is minimal overlap, and only a small part of the story is included in the model while many parameters in the model derive from other sources than the story. This can severely weaken the link between stories and models, and can lead to potentially inconsistent outcomes. More specifically, the weak link between the qualitative and quantitative scenarios is the most problematic aspect of the Story-andSimulation approach (Kok and Van Delden 2009). It limits the integration especially with respect to social (and hard to quantify) data, and strengthening this link is the key challenge in scenario development.

Such shortcomings warrant the development of a portfolio of tools that are tailored to the relevant parties involved. The portfolio approach values and maximises complementarities of varying modelling approaches and analyses across the various aspects of land change (Young et al. 2006). It utilises several types of analysis simultaneously. For example, statistical analyses delineate the forms of relationships in simulation models; narratives help interpret quantitative results; meta-analyses of various case studies produce higher levels of generalisation; and agent-based simulations examine behaviours of key agents at finer scales. The goal is not to identify a single best method for modelling and analysis. It aims to use multiple methods to compare, triangulate and generate a more comprehen- 
sive picture of the land change research in question. Dilemmas remain with the question of balance between diversity and integration. One is that the use of a portfolio approach is in the early stages, and criteria and standards for the 'right' combination have yet to be generated. A second issue is an absence of measuring the added value of new tools and methods introduced to solve some of the balancing issues discussed here.

\section{Conclusion}

Complementarity rather than integration of mixed methods remains the trend du jour in land change science. Geographic information approaches are uniquely positioned to facilitate integration as several applications exist to integrate qualitative and quantitative data in GIS frameworks. You can click on a point, for example, and have qualitative information emerge from that point. This is quite user friendly and visibly demonstrates the integration unlike in other disciplines. However, what remains a challenge is to move from point-sourced qualitative data or 'comments' towards a synergistic process of integrating in-depth and analytical qualitative data that can be translated quantitatively and interpreting such quantitative data qualitatively for policy formulation and academic studies.

Tools and methods are being developed at a dizzying pace and enable the successful advancement of integration and synthesis across land change processes. The use of semi-quantitative approaches such as fuzzy sets and fuzzy cognitive mapping seem promising, as do agent-based models, which better represent the actors and social processes in a land-use system and can link to spatial patterns of land cover, but also require significantly more data inputs and computational power than earlier empirical land-use models.

In addition to developing new methods for integration, a longer-term solution may be to respect the interdisciplinarity of land change research, and understand its underlying systems perspective. This is particularly needed given the disciplinary and therefore epistemological gap between social, economic and actor-oriented land-use research, and biophysically driven land-cover studies. The systems-based interdisciplinary approach can enhance engagement among experts of different methodologies and epistemologies and facilitate shared understanding and implementation of mixed meth- ods. For example, a scenario-based approach includes a portfolio of methods to (1) directly involve a variety of stakeholders in the discussion of the socio-economic context; (2) link this information to quantitative data and models; and (3) provide quantitative, spatially explicit information on current and future patterns of land-use change. This process makes possible exchanges of stakeholder perspectives and expert opinions integrated by fortifying the linkages between (1) and (3), and increases diversity in representation.

\section{Acknowledgements}

So-Min Cheong extends her gratitude to Hal Mooney and Eric Lambin for their inspiration and feedback at the formulating stage of the paper. Dan Brown gratefully acknowledges funding support for research contributing to the ideas presented here from the NSF (CNH\# GEO-0814542) and David Lopez-Carr from the NSF (CNH-EF 070962 BCS-0525592) and NIH K01 (HD049008).

\section{References}

Alcamo J ed 2009 Environmental futures: the practice of environmental scenario analysis Developments in integrated environmental assessment 2 Elsevier, Amsterdam

An L, Linderman M, Qi J, Shortridge A and Liu J 2005 Exploring complexity in a human-environment system: an agent-based spatial model for multidisciplinary and multiscale integration Annals of Association of American Geographers 95 54-7

Carr D L 2008 Migration to the Maya Biosphere Reserve, Guatemala: why place matters Human Organization 67 37-48

D'Aquino P, Le Page C, Bousquet F and Bah A 2003 Using self-designed role-playing games and a multi-agent system to empower a local decision-making process for land use management: the Self Cormas experiment in Senegal Journal of Artificial Societies and Social Simulation (Available at http://jasss.soc.surrey.ac.uk/6/3/5.html) Accessed 7 October 2011

Dearing J, Battarbee R, Dikau R, Larocque I and Oldfield F 2006 Human-environment interactions: towards synthesis and simulation Regional Environmental Change 6 115-23

Gimblett R, Daniel T, Cherry S and Meitner M 2001 The simulation and visualization of complex human-environment interactions Landscape and Urban Planning 54 63-78

Gutman G, Moran E, Rindfuss R, Cochrane M A and Skole D eds 2004 Land change science: observing, monitoring and understanding trajectories of change on the earth's surface Kluwer Academic, Dordrecht

Guyot P and Shinichi H 2006 Agent-based participatory simulations: merging multi-agent systems and role-playing 
games Journal of Artificial Societies and Social Simulation (Available at http://jasss.soc.surrey.ac.uk/9/4/8.html) Accessed 7 October 2011

Kok K and Van Delden H 2009 Combining two approaches of integrated scenario development to combat desertification in the Guadalentín watershed, Spain Environment and Planning B 36 49-66

Kok K, Verburg P H and Veldkamp A 2007 Integrated assessment of the land system: the future of land use Land Use Policy 24 517-20

Lambin E and Meyfroidt P 2010 Land use transitions: socioecological feedback versus socio-economic change Land Use Policy 27 108-18

Moran E F and Ostrom E eds 2005 Seeing the forest for the trees: human environment interactions in forest ecosystems MIT Press, Cambridge MA

Norton B 2005 Sustainability: a philosophy of adaptive ecosystem management University of Chicago Press, Chicago IL

Oreskes N, Shrader-Frechette K and Belitz K 1994 Verification, validation, and confirmation of numerical models in the earth sciences Science 263 641-6

Parker D, Manson S, Janssen M, Hoffmann M and Deadman P 2003 Multi-agent systems for the simulation of land-use and land-cover change: a review Annals of the Association of American Geographers 93 314-37
Pilkey O H and Pilkey-Jarvis L 2007 Useless arithmetic: why environmental scientists can't predict the future Columbia University Press, New York

Rindfuss R, Walsh S, Turner B L, Fox J and Mishra V 2004 Developing a science of land change: challenges and methodological issues Proceedings for the National Academy of Sciences 101 13976-81

Robinson D T, Brown D G, Parker D C, Schreinemachers P, Janssen M A, Huigen M, Wittmer H, Gotts $N$, Promburom P, Irwin E, Berger T, Gatzweiler F and Barnaud C 2007 Comparison of empirical methods for building agent-based models of land and resource use Journal of Land Use Science 2 31-55

Smajgl A, Brown D G, Valbuena D and Huigen M G A 2011 Empirical characterisation of agent behaviours in socio-ecological systems Environmental Modelling and Software 26837 44

Young O R, Lambin E F, Alcock F, Haberl H, Karlsson S I, McConnell W J, Myint T, Pahl-Wostl C, Polsky C, Ramakrishnan P, Schroeder $\mathbf{H}$, Scouvart $\mathbf{M}$ and Verburg $\mathbf{P}$ H 2006 A portfolio approach to analyzing complex humanenvironment interactions: institutions and land change Ecology and Society 1131 (Available at http://www.ecolog yandsociety.org/vol11/iss2/art31/) Accessed 7 October 2011 